does not predissociate in the excited ${ }^{2} \Delta$ states. The ${ }^{2} \Delta$-levels up to $B^{\prime 2} \Delta(v=10)$ must have normal lifetimes. At high resolution the $B^{\prime}-X$-bands $v^{\prime} \geq 3$ prove to be of high absorption intensity; their $f$-values, however, have not yet been measured. The $F-X$ and $N-X$-bands are weak in comparison. They borrow part of their strength from the $B^{\prime}-X$-bands by virtue of the interaction between the upper states. This can clearly be seen in Figure 1, namely in the $F-X(2,0)$-band.

The bands of Figure 1 might well be of interest for future astrophysical or aeronomical observations in the vacuum ultraviolet with satellite spectrographs. Should NO as an emitting molecule be taken into consideration, the search should primarily concern the bands of Figure 1 and Table 1.

TABLE 1

PrinCIPAL HEADS OF ${ }^{2} \Delta-X^{2} \Pi$-BANDS IN FIG. $1(\AA)$

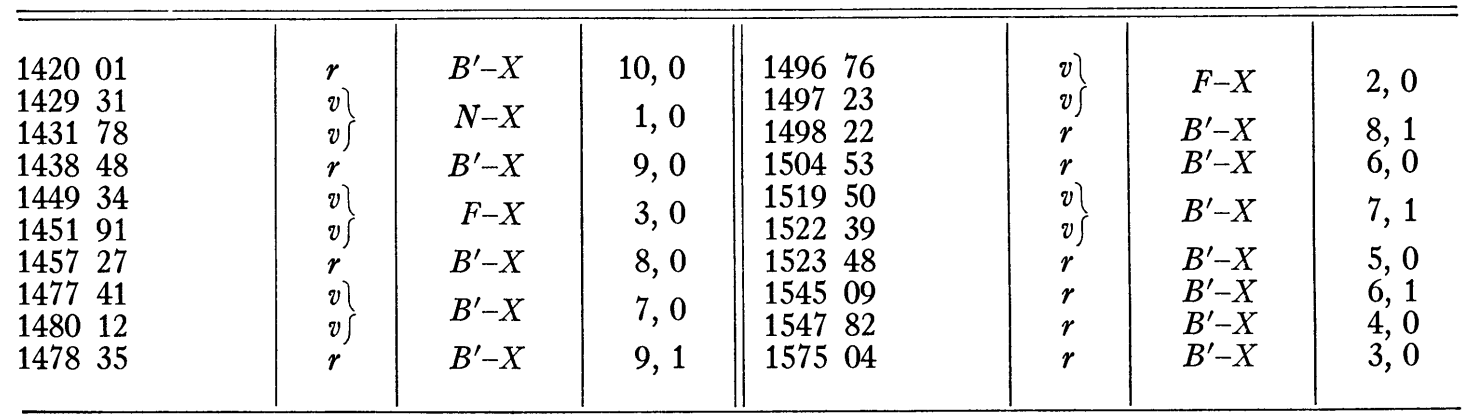

The research was sponsored by the Air Force Cambridge Research Laboratories OAR through the European Office of Aerospace Research, U.S. Air Force, under grant AF-EOAR 63-109.

Ch. Jungen

E. MIESCHER

June 18,1965

Institute of Physics of the University of Basei BASEL, SWITZERLAND

\title{
REFERENCES
}

Baer, P, and Miescher, E. 1951, Helv. Phys Acta, 24, 331. 1953, ibid., 26, 91.

Dressler, K , and Miescher, E. 1965, Ap. J., 141, 1266.

Miescher, E 1955, Canadian $J$ of Phys., 33, 355 . 1956, Helv Phys. Acta, 29, 401 .

Swings, P. 1965, Adv in Space Sci. and Technol, Vol. 7 (New York: Academic Press).

Tanaka, Y. 1953, J. Chem. Phys , 21, 788

\section{SOME ASTRONOMICAL APPLICATIONS OF CROSS-CORRELATION TECHNIQUES*}

Cross-correlation techniques have been used for many years to increase the signal-tonoise ratio of a wide variety of analogue and digital data. A large literature describing these methods exists, and many clever applications have been developed (see Fellgett 1953, and esp. Woodward 1955, for a basic mathematical development and applications).

* Contribution No 1343 of the Division of Geological Sciences, California Institute of Technology, Pasadena, California. 
In general, use of cross-correlation requires that the data be available in digital form, although for some applications specialized analogue equipment may be used (Fellgett 1953). In this Note, we will describe some applications of these techniques to digitized astronomical spectra and suggest some further applications to both digitized and conventionally presented data.

By forming the cross-correlation function

$$
x\left(\lambda^{\prime}\right)=\int_{L} f(\lambda) F\left(\lambda-\lambda^{\prime}\right) d \lambda
$$

from the intensity $f(\lambda)$ along a spectrogram of length $L$ containing grain noise, we may enhance the contrast of lines with a profile $F(\lambda)$. If the expected line profiles are more

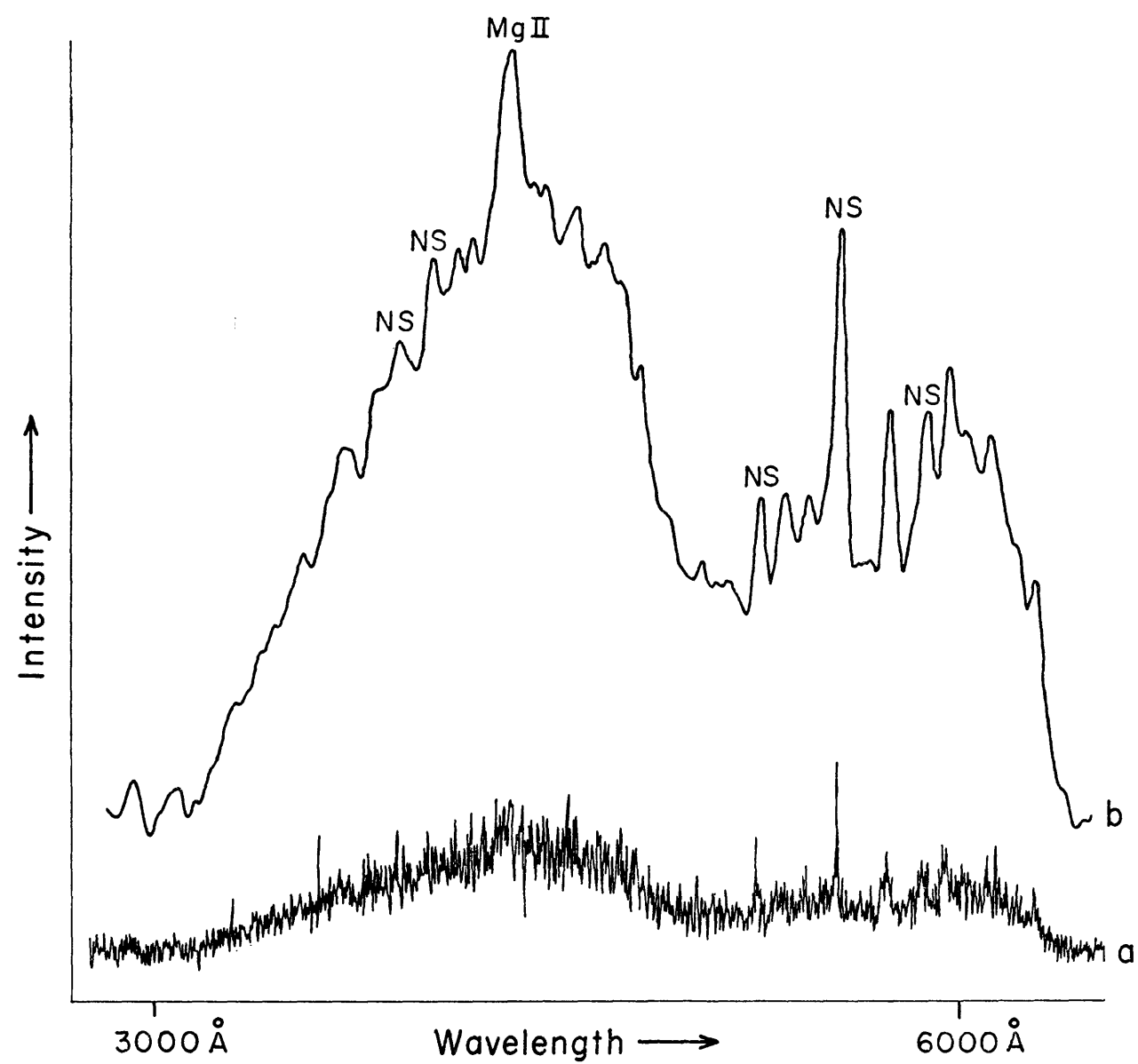

Fig. 1.-(a) Microphotometer tracing of 3C 147 taken by M. Schmidt. (b) Result of cross-correlating with a $15-\AA ̊$ half-width filter function.

narrow than the instrumental profile of the spectrograph, then one should choose $F(\lambda)$ to be the instrumental profile. Alternately, by using a broader $F(\lambda)$ for filtering, one may discriminate against narrow lines and enhance broad features in the spectrum. It is useful to notice that if $F(\lambda)$ is always of one sign, this procedure is analogous to "low-pass filtering" of $f(\lambda)$. Thus, all features broader than $F(\lambda)$ are displayed unalternated.

Figure 1, $a$, illustrates the digitized and intensity-rectified output of a microphotometer tracing of a plate taken by M. Schmidt of the quasi-stellar radio source 3C 147 with the prime-focus nebular spectrograph of the 200-inch Hale telescope. The slit 
width of the microphotometer was about $\frac{1}{10}$ of the plate resolution and was as long as the spectrum width allowed.

Figure 1, $b$, is the cross-correlated output from the input data of Figure 1, $a$, utilizing a Gaussian line profile of about $15-\AA$ half-width. The lines labeled "NS" are night-sky lines identified from a portion of the plate exposed only to the sky. The broad line, identified as Mg II, as well as those previously identified by Schmidt and Matthews (1964), was found on all of seven available plates by this technique. A comparison between these spectra of $3 \mathrm{C} 147$, treated by this filtering technique, indicates that almost all the small features reproduce.

It seems clear that only two or three spectra of this object, treated in this way, would suffice to identify all of the lines normally measured by eye, with perhaps a less subjective result. The procedure seems particularly helpful when broad spectral features are present along with many narrow lines as in the case of $3 \mathrm{C} 147$.

\section{WAVELENGTH MEASUREMENT}

Another application of digital techniques has been an attempt to improve the plateto-plate precision of the wavelength measurements of the hydrogen lines in Sirius B. These lines are very broad, and it is difficult to measure the wavelength accurately by conventional means.

To determine the "center" of a spectral line of known shape, one may cross-correlate the line shape $F\left(\lambda-\lambda^{\prime}\right)$ with the observed spectrum $f(\lambda)$ obtaining a wavelength of maximum correlation, that is, a maximum in the function $x\left(\lambda^{\prime}\right)$. This wavelength corresponds to the "center" of the line if $F\left(\lambda-\lambda^{\prime}\right)$ is a symmetrical function.

In the case of Sirius B, a smooth, symmetrical function was fitted to the observed line profile and cross-correlated with the digitized spectra. Four spectrograms from four separate nights, two in 1961 and two in 1962, were obtained from J. B. Oke, digitized and cross-correlated. Radial velocities calculated from the resultant wavelengths were consistent within a probable error of $\pm 1.6 \mathrm{~km} / \mathrm{sec}$ for four observations. This represents an improvement of about an order of magnitude in the measurement of the radial velocity of Sirius B. A more complete discussion of the observations of Sirius B, including the results of these measurements, is now in preparation.

It should be noted that this cross-correlation technique should be helpful in measuring wavelengths on any plate with broad diffuse lines. However, it will not decrease errors in measurement unrelated to choosing the best "center" of a line (Petrie 1962).

It may also be possible to help "unscramble" blended lines by using special crosscorrelation functions that tend to suppress the lower and the higher spatial frequencies; that is, digital filtering with "band-pass" filters. This technique is presently under study.

\section{ANALOGUE APPROACHES}

Fellgett (1953) has suggested that spatial filtering may be accomplished by analogue techniques in certain cases. For instances, since the exact low-pass cutoff frequency is not too critical, one might use a wide slit on the microphotometer as a low-pass filter. Unfortunately, if the slit is simply widened the spatial filter response is not smoothly cut off but "rings"; that is, it has a $\sin (X) / X$ shape which allows large amounts of highfrequency energy to pass. Rather, one needs a Gaussian-like cutoff which could be produced by a slit with a Gaussian transverse transmission (in the direction of the dispersion of a plate). Unfortunately, since plate transmission is not a linear function of intensity, such a procedure is equivalent to shifting the low-pass cutoff frequency depending on the local plate density. This, for many applications, would nevertheless probably be satisfactory. The use of "zoom" optics in the microphotometer would allow a single Gaussian slit to be used for several different plate dispersions.

The recent development of two-dimensional optical processing using monochromatic 
light and image plane filtering (Jackson 1965) should allow the filtering to be done with the original photographic spectrum in two dimensions. If this technique can be developed, it should be possible to low-pass filter both spectra and conventional astronomical photographs to enhance the visual signal-to-noise ratio. These procedures have the advantage of fast processing and display of the results in a more familiar format as well as considerably increasing the useful information. This optical technique can also be used to enhance fine detail on photographs by selectively filtering out the lower spatial frequencies (Francon 1963). The application of this procedure to lunar and planetary photographs may be quite fruitful.

I would like to thank Drs. J. B. Oke and M. Schmidt for furnishing the plates studied and especially Dr. A. Sandage for his assistance in digitizing the data and evaluating the results, and Dr. G. Neugebauer for valuable suggestions.

J. A. WESTPHAL

May 25, 1965

Division of Geological ScIENCES

California Institute of Technology

PASADENa, CALIFORNia

\section{REFERENCES}

Fellgett, P. 1953, J. Opt. Soc. America, 43, 271.

Francon, M. 1963, Modern Applications of Physical Optics (New York: Interscience Publishers), p. 77. Jackson, P. L. 1965, Appl. Opt., 4, 419.

Petrie, R. M. 1962, Astronomical Techniques, ed W. A. Hiltner (Chicago: University of Chicago Press), p. 63.

Schmidt, M , and Matthews, T. A. 1964, Ap. J , 139, 781.

Woodward, P. M. 1955, Probability and Information Theory, with Applications to Radar (New York: Pergamon Press) 